\title{
Conceptualization of the Life-Span Development Theory: Relevance for Sexual and Reproductive Health in Ghana
}

By

Mavis Dako-Gyeke ${ }^{1 *}, \mathrm{PhD}$ and Baba Musah Iddrisu², MA

\begin{abstract}
Sexual and reproductive health issues continue to generate concerns in contemporary society because it is essential to individuals, couples and families, as well as the social and economic development of communities and nations. The concept of sexual and reproductive health $(\mathrm{SRH})$ provides a comprehensive and integrated approach to health needs related to sexuality and reproduction when viewed from the lens of lifespan development theory. It is a public health concern, human rights issue and is indispensable to the attainment of the Millennium Development Goals because three out of the eight goals are directly related to SRH, while the others have a close relationship with health. The 1994 International Conference on Population and Development in Cairo provided an impetus for program planners, family planning researchers, service providers, policy makers and other stakeholders to recognize the importance of SRH internationally. Nonetheless, the global situation depicts clear inequities between developed and developing regions. Also, women bear the major share of the burden of sexual and reproductive ill health compared to men. It is essential to close these regional and gender gaps since SRH is vital to our quality of life, as other major aspects of health. This paper, which is an exploration of the literature, highlights the (a) importance of SRH across the human lifespan, (b) determinants of SRH in Ghana, and (c) challenges associated with $\mathrm{SRH}$ issues in Ghana. Also, implications of SRH for public health education, policy, practice and research are discussed.
\end{abstract}

Keywords: Development, Ghana, Health, Lifespan, Reproductive, Sexual

\section{Introduction and Background}

Globally, health related issues, particularly sexual and reproductive health concerns need to be addressed in order to achieve sustainable development. This is because sexual and reproductive health ( $\mathrm{SRH})$ is as important to peoples'

\footnotetext{
Department of Social Work, University of Ghana, Legon, Ghana, West-Africa.

${ }^{2}$ University of Bergen, Bergen, Norway.

* Corresponding Author.
} 
quality of life as other key aspects of health, such as eating, sleeping and physical activity (Health Canada, 1999). This is true regardless of age, gender, culture, abilities, sexual orientation, or other characteristics that make up peoples' identity (Health Canada, 1999). Since both female and male sexual and reproductive illhealth concerns threaten international development targets (Institute of Development Studies, 2011), these concerns have increasingly become part of international and national development discourses (Roudi-Fahimi \& Ashford, 2008). Accordingly, there is pressing need for development experts and the international community to recognize the importance of sexual and reproductive health for improving well-being and achieving other social and development goals.

Throughout history sexuality and reproduction have been vital aspects of personal identity and the creation of fulfilling personal and social relationships (Bernstein \& Hansen, 2006). However, the 1994 Cairo International Conference on Population and Development (ICPD) marked a major breakthrough for sexual and reproductive health in international economic and social development circles. At the conference, reproductive health was defined as "a state of complete physical mental and social well-being and not merely the absence of disease or infirmity in all matters related to the reproductive tract, its functions and processes" (World Health Organization, WHO, 2002). The Cairo definition was internationally accepted because it recognized that SRH affects and is affected by the economic circumstances, education, employment opportunities, family structures, as well as political, religious and legal environment (RoudiFahimi \& Ashford, 2008).

Sexual health is the experience of the ongoing process of physical, emotional and sociocultural well-being as it relates to sexuality (The Pan American Health Organization and WHO, 2001). Sexual and reproductive health therefore implies that people are able to have a satisfying and safe sex life, have the capability to reproduce and the freedom to decide if, when and how often to do so (Odoi-Agyarko, 2003; Roudi-Fahimi \& Ashford, 2008). Although Ghana formulated her first national population policy in 1969, the conference paved the way for the policy to be revised to incorporate more comprehensive issues (Odoi-Agyarko, 2003). In 1996, the first national reproductive health policy was formulated in Ghana to provide the framework for guiding reform and development in the provision of sexual and reproductive health services (OdoiAgyarko, 2003). Also, the policy aimed at (a) making reproductive health services accessible and affordable and (b) clarifying the roles of various agencies involved in the provision and financing of reproductive health services.

Currently, the Millennium Development Goals (MDGs) agenda broadly recognizes the importance of sexual and reproductive health issues. Three of the eight goals are directly linked to sexual and reproductive health issues (reduce 
child mortality, improve maternal health, and combat HIV/AIDS, malaria, and other diseases) and the others have a close relationship with health (Fathalla \& Fathalla, 2008; WHO, 2008). Sexual and reproductive health is a broad concept about health, as well as well-being in issues regarding sexual relations, pregnancies, and births (Roudi-Fahimi \& Ashford, 2008). Over the years, in the developed world, improvements have been made in the area of sexual and reproductive health, particularly, contraceptive use (Chaya, Johnston, Engelman, Ethelston \& Greene, 2001). However, in developing countries, most married women do not have access to modern contraceptives and millions of women and adolescent girls persistently suffer from death and disabilities during pregnancy and childbirth (United Nations Population Fund, UNPF, 2008).

\section{Statement of the Problem}

Even though there are unacceptable discrepancies in the sexual and reproductive health status of the populations in Western, Central and Eastern Europe (WHO, 2001), the developing world records the greatest number of these challenges (Gribble \& Haffey, 2008). In developing countries, the majority of impoverished women suffer disproportionately from unintended pregnancies, maternal death and disability, sexually transmitted infections including HIV, gender-based violence and other problems related to their reproductive system and sexual behaviour (United Nations Population Fund, 2008). Whereas there are dramatic improvements in reproductive health in the more developed countries, in developing countries, including Ghana, the situation is different. In the year 2000, ninety-eight percent of the 3.43 million adult deaths from causes linked to poor reproductive health occurred in the developing countries (Chaya et al., 2001). In most developed countries, women have a one in 7,300 lifetime risk of maternal death, compared to a risk of one in 22 for women in sub-Saharan Africa (Gribble\& Haffey, 2008; WHO, 2007).

In the developing world, about 45 million women do not receive high quality prenatal care and 60 million births take place without assistance from skilled health personnel (Chaya et al., 2001). As a result, in sub-Saharan Africa, a maternal mortality ratio of 900 (maternal deaths per 100,000 live births) was the highest in the World Health Organization's estimates published in 2005 (Gribble \& Haffey, 2008). The maternal mortality ratio is high in this part of the world because the majority of victims reside in rural and remote areas where most often pregnancies are unplanned and of high-risk. Abortion is another major sexual and reproductive health issue that poses serious risks to females worldwide because globally, more than one-fifth of the estimated 46 million annual pregnancies are terminated each year (Population Action International, 2001). Twenty million of these abortions are carried out illegally and most often under unsafe conditions 
because majority of these women tend to ignore the legal status or conditions of abortion and therefore are willing to risk unsafe abortions (Chaya et al., 2001).

Compared to the developed world where one in every 85,000 abortions results in death, in Africa, one in every 150 abortions results in death (Chaya et al., 2001). While family planning reduces fertility, prevents mother-to-child transmission of HIV and AIDS and contributes to the overall development (UNAIDS/WHO, 2008) of nations all over the world, in sub-Saharan Africa, only about 23 percent of married women use family planning (Gribble \& Haffey, 2008). The relatively low contraceptive use in addition to high level of unmet family planning needs have resulted in large family sizes with an average of 5.4, 5.7, and 5.5 children for women in sub-Saharan, Western and Eastern Africa respectively, in their lifetime (Gribble \& Haffey, 2008). Large family sizes have negative effects on development targets in a resource constrained continent like Africa. Compared to poor and large families, people who have smaller, as well as healthier families tend to have higher productivity and savings and are better able to escape the vicious cycle of poverty (Roudi-Fahimi \& Ashford, 2008).

The influence of cultural practices and beliefs cannot be ignored in discussions on sexual and reproductive health because cultural taboos are major obstacles to informed discussions and access to sexual and reproductive health services, especially among adolescents in some parts of the world. In Ghana, for instance, it is often difficult for adolescents to discuss sexual and reproductive health issues with adults, particularly their parents due to fear of being seen as engaging in sexual activities. Roudi-Fahimi and Ashford (2008) argue that it is difficult to research on issues related to sexuality and reproduction with particular reference to the Middle East and North Africa because it touches on the private and intimate lives of people. Generally, sexual and reproductive health concerns are sensitive and important issues that affect individuals, couples and communities (Bernstein \& Hansen, 2006). Culturally accepted norms and practices limit females' ability to safeguard their own sexual and reproductive health and survival (Centre for the Study of Adolescence and Population Action International, 2009).

Additionally, more than one million girls in 30 countries still undergo harmful traditional practices, including female genital mutilation (UNAIDS/WHO, 2008). The culture of silence that surrounds sexual and reproductive health in Africa, including Ghana, prevents people from seeking information and care. It also limits governments in their efforts in prioritizing these issues in their development agendas (Roudi-Fahimi \& Ashford, 2008). Since the challenges associated with healthy sexuality and reproduction are severe in developing countries including Ghana, this paper which is an exploration of the literature, discusses the (a) importance of sexual and reproductive health in Ghana across the human lifespan, (b) determinants of sexual and reproductive 
health in Ghana, and (c) challenges associated with sexual and reproductive health issues in Ghana.

\section{The Lifespan Development Perspective}

Although there are numerous perspectives on human growth and development, the lifespan perspective is appropriate for this discussion because sexual and productive health issues influence the development of both sexes from infancy to old age. The proponents of the lifespan theory (e.g., Baltes, Linderberger \& Staudinger, 1998; Berk, 2007) assert that each period of life has its own developmental challenges and accomplishments, and adaptive processes are at work at each of the periods of the life span. No age or stage of development is supreme; rather, events occurring at each stage can have profound effects on future development (Berk, 2007). For instance, in many cultures, discrimination against females that begins in infancy and male attitudes towards gender and sexual relations, which start in boyhood, can determine the course, as well as influence sexual and reproductive health (UNFPA, 2008) of individuals in later years of life.

The lifespan theory also emphasizes that every stage of development has its own agenda, demands and opportunities that yield some similarities in development among individuals in that stage (Berk, 2007). Baltes and colleagues (1998) thus argue that the theory is concerned with comparing an individual's development with that of others and with the individual's own status at various points in time. Human development scholars, such as Berk (2007) and Staudinger and Lindenberger (2003) contend that the lifespan paradigm is built on four important assumptions, which suggest that human development is (a) lifelong, (b) multidimensional or multidirectional, (c) highly plastic, and (d) affected by multiple interacting forces. Human development is life-long because development does not cease when adulthood is reached, but rather it continues till death. Multidimensionality or multidirectional implies that human development is affected by a complex combination of biological, psychological and social forces.

The plasticity of human development signifies how change is possible at all stages as humans grow and mature. However, development gradually becomes less plastic, as capacities and opportunities for change are limited. Human development is influenced by multiple interacting forces because pathways of change are varied and the developmental domains are not separate but overlapping. Nevertheless, individual agency influences the interacting forces across the lifespan because the active and goal-oriented role of individuals in their own development is fundamental. Thus, individuals have to adjust to, cope with, as well as take advantage of the changing opportunities and constraints that 
characterise each stage of development (Heckhausen, Wrosch \& Schulz, 2010). Figure 1 depicts sexual and reproductive health across human lifespan. Throughout their lives, individuals are sexual beings (Health Canada, 1999).

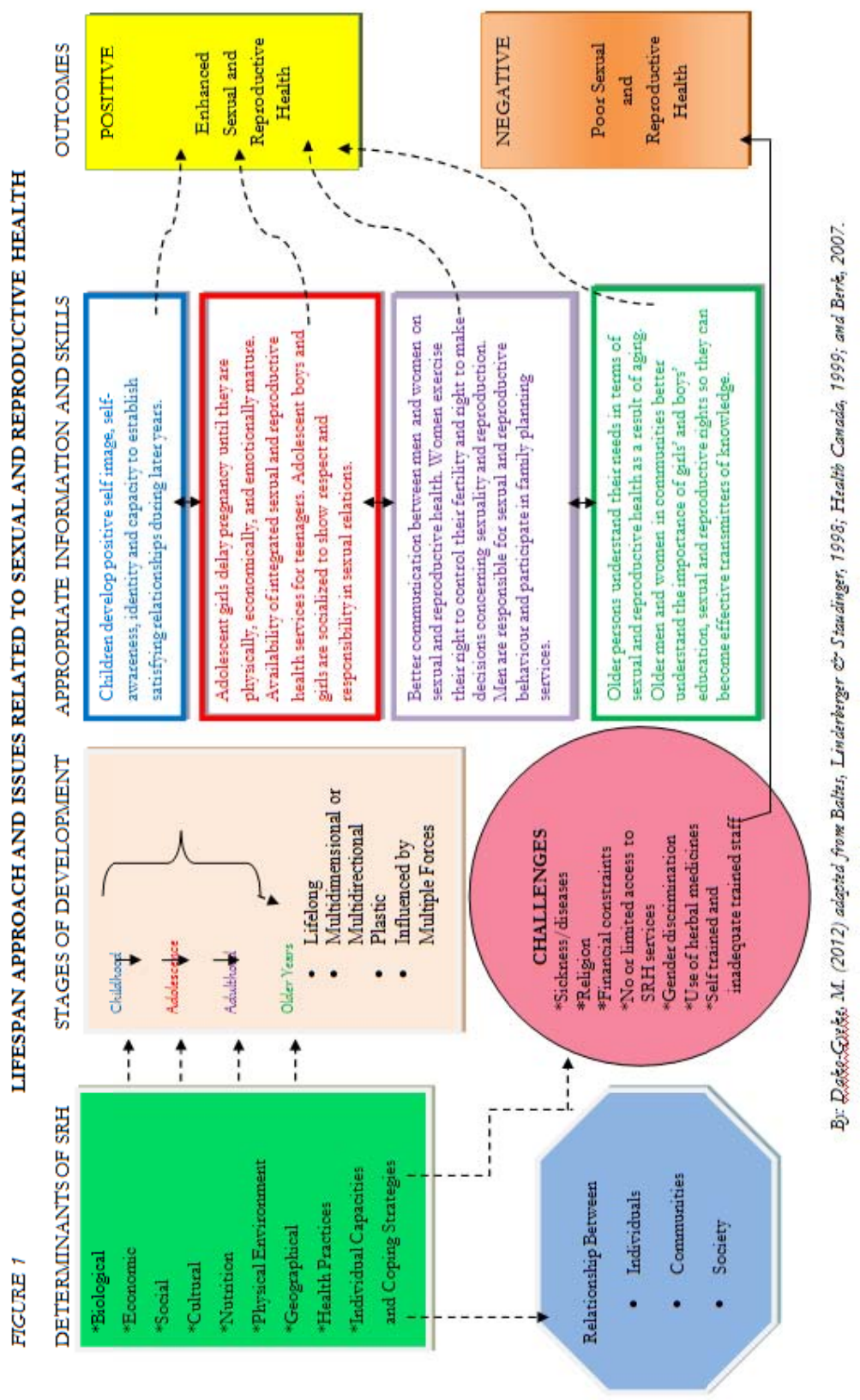


As Figure 1 illustrates, sexual and reproductive health concerns do not only occur during the reproductive years but throughout life. Additionally, SRH health is influenced by varied factors or multiple forces, including biological, economic, social, cultural, physical environment, geographical location, health practices, as well as individual capacities and coping strategies (Health Canada, 1999). These factors affect the relationship between individuals, communities and society and the relationship may bring about challenges. If the challenges are not well managed, they could negatively affect the SRH of people. On the other hand, if individuals are offered appropriate information and are assisted to develop appropriate skills at the various stages of development, their SRH will be enhanced.

\subsection{Determinants of Sexual and Reproductive Health}

Biological make-up is very important because sexual and reproductive activities differ in important respects between males and females (Hansen, Mann, Wong \& McMahon, n. d.). For instance, women are at a greater risk of acquiring certain sexually transmitted diseases due to the greater vulnerability of the female reproductive tract. In Ghana, menopause represents a significant change in most women's sexual and reproductive life biologically, socially and emotionally due to lack of information and societal beliefs. In some regions, menopausal women are often neglected by their husbands or branded as witches and sent to witches camps. The Health Canada's 1999 report on sexual and reproductive health indicates that a wide range of social, economic and cultural factors influence sexual and reproductive health of individuals. The range includes income, social status and hierarchy, social support, beliefs, education, employment and working conditions.

Research has shown that early initiation into sexual activity and riskier sexual practices are higher among youth in lower socio-economic environments (Health Canada, 1999). Globally, education influences decisions and choices regarding sexual and reproductive health because it encourages contraceptive use and promotes postponement of the onset of sexual activity and childbirth, especially among the youth. For example, most often, illiterate or poor women may find it difficult to ask their partners to use condoms. Also, social status determines the degree of control people have over their lives and that of others, especially in marital relationships. As Chaya and colleagues (2001) contend, the low socioeconomic status of women and girls sets the stage for poor sexuality and reproduction. Also, gender inequalities render women more vulnerable because most women in Africa are less likely to ask their partners or husbands to use condoms (Garcia-Moreno \& Watts, 2002; Health Canada, 1999; World Health Organization, 2004). 
Within a given country, wealthier women are more likely to use modern family planning methods than poorer women (Gribble \& Haffey, 2008). The Health Canada (1999) report notes that unemployment and lower status in the workplace can also affect sexual and reproductive health of people. Poor working conditions, such as shorter pregnancy leaves may lead to harmful choices and exposure to hazards. However, social support provided by families, friends, communities, and society in general has beneficial effects on sexual and reproductive health (Health Canada, 1999). The physical environment (both natural and man-made) including housing, quality of air and water, as well as the safety of communities have a major impact on sexual and reproductive health (Health Canada, 1999).

The majority of the products and chemicals, especially alternative or herbal medications used and consumed, significantly affect the SRH of most people because these medications may not be safe and effective. In their study on Ghana's herbal market, van Andel, Myren and van Onselen (2012) found that people preferred herbal medicine for diseases related to SRH. The authors further noted that the most salient application for herbal medicine was women's health, which included plants for strengthening pregnant women, female infertility, abortion and treatment of sexually transmitted diseases (van Andel et al., 2012). Also, drugs and devices related to sexuality and reproduction are part of the physical environment that impact on the SRH of people. For example, the availability and use of birth control pills for contraception, the use of condoms and other family planning devices is very low among people who are not educated and those who live in the rural areas across the world.

The way of life of a people, especially their values, attitudes, and expectations shape their ideas about sexuality, sexual behaviour, as well as their views and choices about reproduction (Health Canada, 1999). Individual capacities and coping skills, such as sense of control, self-esteem and competence are connected to SRH (Health Canada, 1999). Since each individual is unique, people are predisposed to and respond to SRH issues differently. For instance, some genetic deformities or illness may negatively affect SRH of people. In some countries women hold less power in heterosexual relationships and this affects their ability to delay sexual activity, to insist on protection during sexual contact, or to have pleasure in sexual relationships (Health Canada, 1999). Most women are socialized to assume a submissive or passive role and may not have the power to insist on safer sex practices.

Geographical location is another key determinant of sexual and reproductive health. In Ghana, for instance, impoverished women, especially those residing in rural areas suffer disproportionately from unintended pregnancies, maternal death and disability, sexually transmitted infections including HIV, gender-based violence and other problems related to their 
reproductive system and sexual behaviour. Since these women do not have access to quality health services there is a high prevalence of self medication, especially the use of herbal or traditional medications. This practice could result in resistance to prescribed medicines by doctors and could adversely affect the SRH of the people. These harmful and ineffective health care interventions cause harm and waste resources. However, access to safe, effective, and appropriate health services, when they are needed, has a positive influence on SRH. These include services to promote, protect, preserve, and restore SRH (Health Canada, 1999).

\subsection{Experiences of Sexual and Reproductive Health at the Various Stages of Development}

The determinants (Figure 1) affect all the stages of development, and could lead to healthier sexuality and reproduction if they do not become challenges. However, individuals, families, communities could experience poor sexual and reproductive health if possible challenges which may arise are not addressed. During childhood, healthy sexual development, including development of intimacy and trust, gender identity and positive experience of sensual and sexual feelings begin (Hansen et al., n. d.; Health Canada, 1999). Children who develop these characteristics are likely to have a healthy sexuality and better decision making regarding sexuality during adolescence (Figure 1).

At the childhood stage, the development of a healthy sexuality gives children the ability to establish a positive self image and self-awareness, which are prerequisites for establishing satisfying relationships during later years of life (Health Canada, 1999). However, vulnerable children, especially those on the streets, may not be able to develop these characteristics and may be pushed into prostitution at an earlier age or end up becoming pregnant. In addition, children who experience harmful cultural practices like female genital mutilation may have sexual and reproductive health problems during their later years of life.

The adolescence stage is very crucial because high teenage fertility rate is a global public health concern. Adolescent fertility has economic and health consequences because research shows that early childbearing hinder the economic and educational opportunities of adolescent mothers and their children (Population Action International, 2001). Adolescents face risks related to early sexual experience, marriage and child bearing (UN Millennium Project, 2006). Since it is a stage at which individuals attain physical, sexual and social maturity, adolescents are normally socialized into adult roles. However, developing attitudes about sexuality and reproduction are strongly affected by several variables, such as peer relationships, school environment, family circumstances, as well as personal vulnerability. This could pose a challenge, especially for adolescent girls who may be at risk of (a) being infected by sexually transmitted 
diseases, including HIV and AIDS (WHO, 2001), (b) engaging in prostitution or (c) becoming teenage mothers.

Availability of appropriate knowledge and services on SRH health that is tailored to the needs of adolescents will help adolescent girls to stay in school and delay pregnancy until they are physically, economically and emotionally mature and consequently improve their sexual and reproductive health (Figure 1). On the other hand, adolescents who do not have information on or access to SRH services, especially those residing in rural or deprived areas of Ghana may engage in unprotected sex, drop out of school, experiment with other sexual practices or become teenage mothers. The 2008 Ghana Demographic Health Survey indicates that by region, the percentage of females aged 15-19 who had begun childbearing ranged from seven percent each in the Western and Greater Accra regions to twenty-three percent in the Central and Northern regions (Ghana Statistical Service \& Ghana Health Service, 2009). Early pregnancy and childbirth could negatively affect the health, as well as the future socioeconomic prospects of both the teenagers and their children.

For most people, dealing with conditions and diseases that unfavourably affect sexual and reproductive health are essential during adulthood because at that stage sexual activity, reproduction, and parenthood become very important (Health Canada, 1999). Sexual feelings and expression, sexual decision-making, longer-term commitment to a partner, decision on whether and when to have children, pregnancy and childbirth, contraceptive use, and protection from sexually transmitted diseases, including HIVand AIDS become issues of concern at this stage of development (Health Canada, 1999). In addition to these, social expectations about gender roles and power affect SRH choices. In Ghana for example, although both men and women could have problems associated with fertility, most often females are held responsible for infertility. Consequently, most men do not avail themselves for treatment of fertility related diseases.

From childhood, most boys are socialized to view reproductive health issues as feminine. It is therefore important that during the earlier years of life (childhood and adolescence), both girls and boys are provided with appropriate sexual and reproductive information and skills (Figure 1). Even during adulthood, lack of information and access to sexual and reproductive health services due to financial constraints, lack of education or geographical location can be obstacles to improved SRH. For example, the 2008 Ghana Demographic and Health Survey indicated that in the poorest regions of Ghana, the Central region in the south, the Northern and Upper West regions in the North, recorded the highest rates of infant mortality in the country (Ghana Statistical Service \& Ghana Health Service, 2009). The survey also showed that under-five mortality ranged from a low of 50 deaths per 1,000 live births in the Greater Accra and Volta regions to a high of 142 and 137 deaths per 1,000 live births in the Upper West and the 
Northern regions, respectively (Ghana Statistical Service \& Ghana Health Service, 2009).

Sexual feelings and responses continue into older years of life although sexual expressions may change with physical and emotional effects of aging. In most societies in the developing world including Ghana, the majority of older persons, especially women, do not understand the changes that occur due to aging, such as menopause. Also, while it is normal for older men to express their sexual feelings, generally, older women are regarded as asexual and are therefore stigmatized and discriminated against when they engage in sexual activities (Owusu \& Anarfi, 2010). However, older women who are knowledgeable about their sexual health would be able to stand up for their rights and advocate for other older women who do not have much information (Figure 1). This is important because the plasticity of human development clearly indicates that aging is normal. In this regard, both women and men should be allowed to express their sexual feelings during older years since this has implications for their health and well-being.

\section{Sexual and Reproductive Health Challenges in Ghana}

After the 1994 Cairo conference, program planners, family planning researchers, service providers, policy makers and other stakeholders have recognized the importance of sexual and reproductive health globally (Blanc, 2001). However, there is mounting concern about sexual and reproductive health challenges in the developing world (Gribble \& Haffey, 2008; Mayhew \& Adjei, 2004), including Ghana where there are physical, social and financial barriers. While sexual and reproductive health programmes are a key feature of the Ghana Ministry of Health's programmes, the task of measuring it is complex in view of the fact that many health conditions are indirectly linked to sex and reproduction (Adanu, Seffah, Anarfi, Lince, \& Blanchard, 2012).

Given that sexual and reproductive health touches on sensitive religious, cultural, and social aspects of people's lives in Ghana (Tulloch et al, 2011), many people feel uncomfortable or embarrassed when discussing issues related to it. Montagu and Graff (2010) concur and argue that SRH issues bring about an emotional response that ignites unique cultural and moral debates. In this regard, the majority people with sexually transmitted infection symptoms tend not to report their symptoms to professional health workers (Adanu et al., 2008).This could lead to reproductive health problems, like infertility which has major social consequences for married couples, especially Ghanaian women (Adanu et al., 2012). Additionally, findings from studies show that although most Ghanaian women have a very high level of knowledge about modern contraception, their knowledge does not translate into use (Adanu et al., 2009; Adanu et al., 2012) 
probably because these are decisions women have to take in consultation with their male partners. It could also mean that most women still have inaccurate information about how these methods work and the possible side effects (Adanu et al., 2012).

Some scholars (e.g., Adongo et al., 1997; Adongo, Phillips, \& Binka, 1998b; Mayhew \& Adjei, 2004; Parr 2002) have argued that reasons for the continuing lack of uptake of family planning services are not well understood though could include socio-cultural factors, poor access for adolescents and unmarried women, and continuing quality of care issues. The influence of culture is entrenched in the sexual and reproductive health practices of most Ghanaians, especially those residing in rural areas. For instance, while efforts to eradicate female genital mutilation are ongoing, the results are still modest due to deeprooted cultural practices that expose most females to serious health consequences (Sakeah, Beke, Doctor \& Hodgson, 2006). In their study on males' preference for circumcised women in Northern Ghana, Sekah et al. (2006) found that ethnicity and religion were significant factors that influenced males' preference to marry circumcised women.

Furthermore, although SRH data generated in various departments of the Ghana Health Service and the university system have improved over the years, obviously there is lack of information on SRH that are not national priority areas (Mayhew \& Adjei, 2004). Particularly, there are no accurate data on reproductive cancers, infertility, gender-based violence, female genital mutilation, safe abortion and post-abortion care services and STIs (Adongo, Akweongo, Binka, \& Mbacke, 1998a; Mayhew \& Adjei, 2004; Mbacke, Adongo, \& Akweongo, 1998). These issues may not have received much attention because policy actors who take national priority decisions in Ghana do not have (a) ready access to SRH data and (b) the inclination to address issues that are not included in the Millennium Development Goals (Mayhew \& Adjei, 2004). Also, SRH stakeholders have been slow in tackling these issues because the issues are more controversial (Mayhew \& Adjei, 2004).

Another sexual and reproductive health challenge in Ghana is the increased sexually active behaviour of adolescents as confirmed by the 2008 Ghana Demographic and Health Survey, which indicated that eight percent of females and five percent of males have had sexual intercourse by age 15 . In addition, the survey reported that 63 percent of females and 78 percent of males between ages 15-19 have had sex sexual intercourse (Ghana Statistical Service \& Ghana Health Service, 2009). As Biddlecom, Munthali, Singh and Woog (2007) opined, many young people in sub-Saharan Africa face the risk of HIV, sexuallytransmitted infections and unintended pregnancies. This notwithstanding, pregnancy and sexually transmitted infection (STI) prevention and treatment services and HIV testing are under-utilized among sexually-active adolescents. 
The majority of sexually-active adolescents are not well prepared to prevent pregnancy since a large proportion of them do not use contraception (Biddlecom et al., 2007).

Also, adolescents may not seek treatment for STIs because of lack of knowledge about places for getting treatment, lack of awareness of STIs or because of perceived barriers in accessing SRH services (Biddlecom et al., 2007). The most common barrier for teenagers to obtaining either contraceptive methods or STI diagnosis and treatment is social stigma in the form of either fear or embarrassment, which may partly explain why their access to reproductive health services are not optimally met (Amuyunzu-Nyamongo, Biddlecom, Ouedraogo \& Woog, 2005; Biddlecom et al., 2007; Dehne \& Riedner, 2008). Furthermore, adolescents may try several means at once or go through sequential steps to treat a health problem through self-medication (Biddlecom et al., 2007) since at this stage of development, most adolescents are adventurous. This may worsen the situation and consequently delay the healing process as most sexuallyactive adolescents are more in need of SRH services given their possible exposure to STI's.

In Ghana, sexual and reproductive health is an area that is dominated by both trained and untrained herbal medicine practitioners, especially in rural and deprived areas. The proliferation of herbal medicine and the treatment of different diseases by a single medical preparation have become a public health concern (van Andela, Myrenb \& van Onselen, 2012). Also, concerns have been raised about the safety and potency of herbal medicines in terms of their preparation, hygiene, dosage of administration and packaging (Ofori, 2011). These notwithstanding, many people still patronize these medicines due to financial constraints, lack of healthcare facilities, religious or cultural beliefs, among others. The cost of services and not knowing where to go are barriers to obtaining SRH services (Biddlecom et al., 2007).

Public financing for sexual and reproductive health services has limited benefits for the poor, especially those in the rural areas of Ghana because it is often directed at services (e.g. sterilization) that are mostly delivered by providers in urban settings serving a higher-income clientele (Montagu \& Graff, 2010). In addition, exclusively subsidizing government or non-governmental delivery channels can also exclude adolescents and other populations who may prefer to seek SRH services from private providers (Decker \& Montagu, 2007; Murray, Dougherty, Stewart, Buek \& Chatterji, 2003). For example, treatment of STIs, abortion services and normal deliveries are more likely to be delivered by private providers who are not subsidized by the government, and for whom government oversight of quality is lenient or non-existent (Montagu \& Graff, 2010).

According to Tulloch et al (2011), the often highly politicised nature of sexual and reproductive health issues obscures the factors that influence policy 
development and implementation. The influence of politics in funding priorities is particularly evident in countries like Ghana where there are considerable differences in sources of SRH services between urban and rural populations (Montagu \& Graff, 2010). In rural areas, sexual and reproductive health services are most often provided by community health nurses. However, the inefficient deployment of community health nurses failed to increase the coverage of sexual and reproductive health services because resources for posting them to communities were constrained (Awoonor-Williams, Vaughan-Smith \& Phillips, 2010).

Awoonor-Williams and colleagues (2010) further argued that in most underserved rural areas of Ghana, sub-district clinics were not only geographically remote from most households, but clinical services were culturally inappropriate. Since culture plays an important role in peoples' decisions about sexual and reproductive health, services that do not conform to cultural practices may not be utilized even if they are accessible and free. This situation is likely to be prevalent in most rural areas of Ghana, where traditional practices are regarded as sacred, and could be one of the reasons accounting for the differences between SRH service utilization among urban and rural populations.

\section{Conclusions and Implications}

Sexual and reproductive health is vital for the achievement of the Millennium Development Goals (United Nations Population Fund, 2008) because every human being has the right to enjoy healthy sexuality and reproduction. Based on the key tenets of the lifespan developmental perspective, it is obvious that $\mathrm{SRH}$ is a lifetime concern for both females and males. As sexual and reproductive ill-health issues continue to threaten national and global development targets, it is essential to make positive connections between sexual and reproductive health and lifespan development. Without access to comprehensive sexual and reproductive health information and services, people are more likely to have poor SRH, which has implications for global poverty reduction efforts.

The lifespan approach to sexual and reproductive health has implications for public health education, policy, practice and research. Since sexual and reproductive health remains the principal cause of ill health and death of women of childbearing age, especially in developing countries (United Nations Population Fund, 2008), there is urgent need for public health education and service provision for SRH to be strengthened. Additionally, the existence of barriers to accessing SRH services, particularly among adolescents, signifies not 
only the need for age specific interventions, but also interventions that focus on specific geographical areas in Ghana. There is therefore the need for more widespread provision of information on SRH services, as well as on sources from which relevant services may be obtained (Biddlecom et al., 2007). This will ensure that SRH services will be available to neglected subgroups in Ghana.

Furthermore, because healthy sexuality and reproduction is a right for everyone across the lifespan, policy initiatives and programs should be tailored to different challenges faced by both females and males at different stages in life. In view of the fact that majority of research on sexual and reproductive health focuses on the reproductive years of life and on females, there should be more research on males, as well as sexual health during childhood and older years of life. In addition, questions regarding societal knowledge, values and attitudes towards SRH must be raised and addressed as a human rights issue. This will encourage individuals, families, communities and the nation as a whole to be committed to and support sexuality and reproduction as very important aspects of life across the lifespan.

\section{References}

Adanu, R. M., Seffah, J., Anarfi, J. K., Lince, N., \& Blanchard, K. (2012). Sexual and Reproductive health in Accra, Ghana. Ghana Medical Journal, 46(2), 58-65.

Adanu, R. M., Allan, G. H., Sefah, J. D., Darko, R., Anarfi, J. K., \& Duda, R. B. (2008). Sexually Transmitted infections and health seeking behaviour among Ghanaian women in Accra. African Journal of Reproductive Health, 12(3).

Adanu, R. M., Seffah, J. D., Hill, A. G., Darko, R., Duda, R. B., \& Anarfi, J. K. (2009). Contraceptive use by women in Accra, Ghana: Results from the 2003 Accra Women's Health Survey. African Journal of Reproductive Health, 13(1), 123-133.

Adongo, P. B., Phillips, J. F., Kajihara, B., Fayorsey, C., Debpuur, C., \& Binka, F. N. (1997). Cultural factors constraining the introduction of family planning among the KassenaNankana of Northern Ghana. Social Science and Medicine. 45, 1789-804.

Adongo, P., Akweongo, P., Binka, F. N., \& Mbacke, C. (1998a). Female genital mutilation: Socio-cultural factors that influence the practice in Kassena-Nankana District, Ghana. African Journal of Reproductive Health, 2, 25-36.

Adongo, P. B, Phillips, J. F., \& Binka, F. N. (1998b). The influence of traditional religion on fertility regulation among the Kassena- Nankana of Northern Ghana. Studies in Family Planning, 29, 23-40.

Amuyunzu-Nyamongo, M., Biddlecom, A. E., Ouedraogo, C., \& Woog, V. (2005). Qualitative evidence on adolescents' views of sexual and reproductive health in sub Sabaran Africa. Occasional

Report No. 16. New York: Alan Guttmacher Institute.

Awoonor-Williams, J. K., Vaughan-Smith, M. N., \& Phillips, J. F. (2010). Scaling up health system innovations at the community level: a case-study of the Ghana experience. In S. Malarcher (Ed.), (pp. 38-51). Social determinants of sexual and reproductive bealth: Informing future research and programme implementation. Geneva: World Health Organization Press. 
Baltes, P. B., Lindenberger, U., \& Staudinger, U. M. (1998). Life-span theory in developmental psychology. In W. Damon \& R. M. Lerner (Eds.), Handbook of child psychology (pp. 1029_ 1143). New York: Wiley.

Berk, L. E. (2007). Development through the lifespan. Boston: Allyn \& Bacon.

Bernstein, S. \& Hansen, C. J. (2006). Public choices, private decisions: Sexual and reproductive health and the Millennium Development Goals. United Nations Development Programme.

Biddlecom, A. E., Munthali, A., Singh, S., \& Woog, V. (2007). Adolescents' views of and preferences for sexual and reproductive health services in Burkina Faso, Ghana, Malawi andUganda. African Journal of Reproductive Health, 11(3), 1-13.

Blanc, A. K. (2001). The effect of power in sexual relationships on sexual and reproductive health: An examination of the evidence. Studies in Family Planning, 32(3), 189-213.

Centre for the Study of Adolescence and Population Action International. (2009). A for Sub Saharan Africa. CSA and PAI.

Chaya, N., Johnston, B., Engelman, R., Ethelston, S. \& Greene, M. E. (2001). The PAI Report Card. A world of difference: Sexual and reproductive health and risks. Population Action International.

Decker, M., \& Montagu, D. (2007). Reaching youth through franchise clinics: Assessment of Kenyan private sector involvement in youth services. Journal of Adolescent Health, 40, 280-282.

Dehne, K. L., \& Riedner, G. (2005). Sexually transmitted infections among adolescents: The need for adequate health services. Geneva: World Health Organization.

Fathalla, M. F. \& Fathalla, M. F. (2008). Sexual and reproductive health: Overview. Elsevier Inc.

Garcia-Moreno, C. \& Watts, C. (2002). Violence against women: Its importance for HIV/AIDS prevention. AIDS, 24 (3), 253-265.

Ghana Statistical Service and Ghana Health Service. (2009). Ghana demographic and health survey 2008. Retrieved September 15, 2011, from http://pdf.usaid.gov/pdf docs/PNADO176.pdf

Gribble, J. \& Haffey, J. (2008). Reproductive health in Sub-Saharan Africa. Population reference bureau, BRIDGE.

Hansen, L., Mann, J., Wong, T., \& McMahon, S. (n. d.). Sexual health. Women's Health Surveillance Report. Retrieved January 10, 2012, from http://www.phac-aspc.gc.ca/publicat/whsr-rssf/pdf/WHSR Chap 23 e.pdf

Health Canada. (1999). Report from consultations on a framework. for sexual and reproductive health. Health Canada, Ottawa.

Heckhausen, J., Wrosch, C. \& Schulz, R. (2010). A motivational theory of life-span development. Psychological Review, 117(1), 32-60.

Institute of Development Studies. (2011). Sexual and reproductive health and rights. Retrieved August 22, 2011, from http://www.eldis.org/health/srhr.htm

Mayhew, S. H., \& Adjei, S. (2004). Sexual and reproductive health: Challenges for prioritysetting in Ghana's health reforms Health Policy and Planning, 19(1), 150-161.

Mbacke, C., Adongo, P., Akweongo, P., (1998). Prevalence and correlates of female genital mutilation in the Kassena-Nankana District of Northern Ghana. African Journal of Reproductive Health, 2, 13-24.

Montagu, D., \& Graff, M. (2010). Financing mechanisms to improve equity in service delivery. In S. Malarcher (Ed.), (pp. 16-37). Social determinants of sexual and reproductive health: Informing future research and programme implementation. Geneva: World Health Organization Press.

Murray, N. J, Dougherty, L., Stewart, L., Buek, K., \& Chatterji, M. (2003). Are adolescents and young adults more likely than older women to choose commercial and private sector providers of modern contraception? The Futures Group. 
Odoi-Agyarko, H. (2003). Profile of reproductive health situation in Ghana. World Health Organization, Ghana.

Ofori, V. A. (2011). Return to natural living: The role of traditional medical practice in Ghana. Retrieved July 10, 2012, from http:// citifmonline.com/?id=1.671653

Owusu, A. Y. \& and Anarfi, J. K. (2010). Sexuality of the aged and people with disability: Challenging popular folkways. Research Review, 26(1), 61-79.

Parr, N. (2002). Family planning promotion, contraceptive use and fertility decline in Ghana. African Population Studies, 17, 83-101.

Population Action International. (2001). A world of difference and risk: Sexual and reproductive bealth and risks. The PAI report card 2001.

Roudi-Fahimi, F. \& Ashford, L. (2008). Sexual and reproductive health in the Middle East and North Africa: A guide for reporters. Washington, Population Reference Bureau.

Sakeah, E., Beke, A., Doctor, H. V., \& Hodgson, A. V. (2006). Males' preference for circumcised women in Northern Ghana. African Journal of Reproductive Health, 10(2), 37-47.

Staudinger, U. M., \& Lindenberger, U. (2003) (Eds.). Understanding human development: Dialogues with lifespan psychology. USA: Kluwer Academic Publishers.

The Pan American Health Organization and World Health Organization. (2001). Promotion of sexual health: Recommendations for action. Guatemala: World Association for Sexology.

Tulloch, O., Mayaud, P., Adu-Sarkodie, Y., Opoku, B. K., Lithur, N. O., Sickle, E., . . Theobald, S. (2011). Health Research Policy and Systems, 9(1).

UN Millennium Project. (2006). Public choices, private decisions: Sexual and reproductive health and the Millennium Development Goals. United Nations Development Programme.

United Nations International Conference on Population and Development. (1994). Programme of Action. Unites Nations.

United Nations Population Fund. (2008). Retrieved August 22, 2011, from bttp:/ / www.unfpa.org/rh/.

UNAIDS/WHO. (2008). Report on Global HIV/AIDS Epidemic. Geneva, UNAIDS.

United Nations Population Fund. (2008). Improving reproductive health. Retrieved August 22, 2011, from http:// www.unfpa.org/rb/

van Andela, T, Myrenb, B., \& van Onselen, S. (2012). Ghana's herbal market. Journal of Ethnopharmacology. Retrieved August 06, 2012, from http://img.modernghana.com/images/content/report_content/ghanas_herbal_market_v an_andel_et_al_2012.pdf

World Health Organisation. (2001). WHO regional strategy on sexual and reproductive health. Reproductive health/pregnancy programme. Copenhagen, WHO Regional Office for Europe.

World Health Organization. (2002). Defining sexual health. Report of a technical consultation on sexual bealth. Geneva, WHO.

World Health Organization. (2004). Gender dimensions of HIV status disclosure to sexual partners: Rates, barriers and outcomes. Geneva: WHO. Retrieved August 22, 2011, from www.who.int/gender/documents/en/genderdimensions.pdf

World Health Organization. (2007). Maternal mortality in 2005: Estimates developed by WHO, UNICEF, UNFPA, and the World Bank. Geneva: WHO.

World Health Organization. (2008). Providing the foundation for sexual and reproductive health: A record of achievement. Geneva, WHO. 
